\title{
Sulle curve razionali dotate di cuspidi.
}

Nota di Ambrogro Longhi (a Lugano).

Sunto. - Si espongono alcuni risultati di geometria numerativa concernenti le curve razionali iperspaziali.

1. In uno spazio lineare a quante si vogliano dimensioni, sopra una curva razionale e irriducibile $\Gamma$ si consideri una serie algebrica $\gamma_{n}^{r}$, di dimensione $r$, d'ordine $n$ e d'indice $\nu \geq 1$, di gruppi di punti: necessariamente equivalenti per la razionalita di $\Gamma$.

Se $v_{1}, v_{2}, \ldots, v_{t}$ sono $t$ numeri (distinti o no) interi $\theta$ positivi, tali ohe:

$$
v_{1}+v_{2}+\ldots+v_{t}=r
$$

con $1 \leq t \leq n-r$, la $\gamma_{n}^{r}$ possiede in generale un numero finito $N$ di gruppi dotati eiasouno di (l) $t$ punti rispettivamente multipli (1) secondo $v_{i}+1$ $(i=1,2, \ldots, t)$; ed è noto come $N$ si possa calcolare mediante una formula di De Jonquitress, la quale, nel caso della curva sostegno $\Gamma$ razionale, diviene $\left({ }^{2}\right)$ :

$$
N=\frac{t !}{\alpha_{1} ! \alpha_{2} ! \ldots \alpha_{r} !}\left(\nu_{1}+1\right)\left(\nu_{2}+1\right) \ldots\left(\nu_{t}+1\right) \vee\left(\begin{array}{c}
n-r \\
t
\end{array}\right)
$$

supposto che nell'insieme dei $t$ numeri $\gamma_{i}$, riducentisi a $\tau$ distinti, ve ne siano $\alpha_{j}$ eguali fra di loro ma diversi dai rimanenti $t-\alpha_{j}(j=1,2, \ldots, \tau)$

E però da osservare che se la curva $\Gamma$ ha delle cuspidi, nel numero $\mathrm{N}$ fornito dalla (1) risultano conteggiati, in modo affatto incognito, anche $i$ gruppi $d i \gamma_{\mathrm{n}}^{\mathrm{r}}$ con almeno uno dei t punti multipli situato in uno cuspide: mentre in molti importanti problemi occorre fare astrazione da tali gruppi.

Così, ad esempio, supponendo la curva $\Gamma$ una $\Gamma_{n}^{0}$ di ordine $n$, con $\rho$ cu. spidi ordinarie in posizione generica, senza altri elementi stazionari ohe iperpiani ordinari, e appartenente allo spazio $S_{r}$ ad $r$ dimensioni, il numero dei suoi iperpiani $r$-tangenti (ciascuno ad $r$ rami) eguaglia quello dei gruppi

(1) Sottintendasi : almeno e in generale precisamente.

(2) E. DE Jonquíres, Mémoire sur les contacts multiples d'ordre quelconque ... «Jonrnal für Math. », 66, 1866. Cf. pure: F. SeverI, Trattato di geometria algebrica, Vol. I, Parte I (Bologna, 1926), p. 243; R. ToRelli, Dimostrazione di una formula di De Jonquières e suo significato geometrico, «Rendiconti del Circolo Matematico di Palermo», 21, 1906, e Sui sistemi algebrici di curve appartenenti ad una superficie algebrica, Atti dell'Accademia di Torino", 42, 1907, n. 1, ultima nota. 
con $r$ punti doppi tutti distinti dalle $\rho$ cuspidi $\left({ }^{3}\right)$ entro la serie lineare $g_{n}^{r}$ delle sezioni iperpiane di $\Gamma_{n}^{0}$ : onde la formula (1) di De JoNQUirines non $\grave{e}$ atta a determinare il numero suddelto se non quando $\rho=0$. Ponendovi $t=r$, $\nu=1, v_{i}=1, \tau=1, \alpha_{1}=r$ essa offre soltanto il numero:

$$
2 \cdot\left(\begin{array}{c}
n-r \\
r
\end{array}\right)
$$

degli iperpiani $r$-tangenti di una generica $\left({ }^{4}\right)$ curva razionale d'ordine $n$ dello spazio $S_{r}$.

Ben diversa, e assai meno semplice, è l'espressione del numero stesso nel caso $\rho>0$ : vedasi il n. 3 .

2. In un lavoro in corso di pubblicazione $\left(^{5}\right)$ risolvo appunto la questione di precisare il numero $\chi$ dei gruppi della serie $\gamma_{\mathrm{n}}^{\mathrm{r}}$, d'indice $\nu$, dotati ciascuno dei t punti $\left(v_{\mathrm{i}}+1\right)$-upli summenzionati $(\mathrm{n} .1)$ : però tutti distinti dalle $\rho$ cu. spidi della curva sostegno $\Gamma_{\mathrm{n}}^{\circ}$ razionale (n. 1). E dimostro il teorema espresso dalla seguente formula:

$$
\chi=\frac{\gamma}{\alpha_{1} ! \alpha_{2} ! \ldots \alpha_{\tau} !} \sum_{k=0}^{t}(t-k) ! k ! s_{k}\left(\begin{array}{c}
n-r-k \\
t-k
\end{array}\right)\left(\begin{array}{c}
n-r-p \\
k
\end{array}\right),
$$

ove $i \tau$ numeri $\alpha_{j}(\mathrm{j}=1,2, \ldots, \tau)$ sono quelli già definiti nel $\mathrm{n} .1$, mentre $\mathrm{s}_{0}=1$ ed $\mathrm{s}_{\mathrm{k}}(\mathrm{k}=1,2, \ldots, \mathrm{t})$ è la somma dei prodotti $a \mathrm{k} a \mathrm{k}$ dei $\mathrm{t}$ numeri (distinti o no) $v_{1}, v_{2}, \ldots, v_{t}$.

Si avverta che nella (2), e in tutte le formule del seguito, è da ritenere $\left(\begin{array}{l}h \\ 0\end{array}\right)=1$ anche quando $h \leq 0$, ed è $\left(\begin{array}{l}h \\ k\end{array}\right)=0$ solo se $0 \leq h<k$; dovendosi invece porre:

$$
\left(\begin{array}{l}
h \\
k
\end{array}\right)=(-1)^{k}\left(\begin{array}{c}
k-h-1 \\
k
\end{array}\right)
$$

se $h<0<k$.

3. Applicando la formula (2) alla serie lineare staccata sulla curva $\Gamma_{n}^{0}$ di $S_{.}$(n. 1) dagli iperpiani passanti per un generico spazio $S_{r-\sigma-1}$, si perviene al teorema che segue:

Gli iperpiani aventi ciascuno con una curva razionale $\Gamma_{\mathrm{n}}^{0} d^{\prime}$ ordine $\mathrm{n}$,

(3) Se queste non fossero au $\Gamma_{n}^{0}$ in posizione generica, potrebbero eccezionalmente esistere gruppi aventi ad esempio $r-1$ punti doppi in punti semplici di $\Gamma_{p b}^{0}$ e un punto triplo (almeno) in una cuspide: ed allora tali eventuali gruppi sarebbero da conteggiare nel nu. mero di quelli situati in iperpiani $r$-tangenti di $\Gamma_{n}^{0}$.

(4) Cosi, per brevità, si chiamerà la curva $\Gamma_{n}^{0}$, sopra considerata, nell'ipotesi $p=0$.

(5) Nei "Commentarii Math. Helvetici : A. LongHI, I gruppi con elementi multipli distinti dalle cuspidi nelle serie algebriche sulle curve razionali cuspidate. 
appartenente ad $\mathrm{S}_{x}$ e dotata di $\rho$ cuspidi $\left({ }^{\circ}\right)$, t distinti contatti (7) di rispettivi dati ordini $\gamma_{i} \geq 1(i=1,2, \ldots, t)$, con :

$$
\nu_{1}+\nu_{2}+\ldots+\nu_{t}=\sigma \leq s
$$

e $1 \leq \mathrm{t} \leq \mathrm{n}-\sigma$, formano una varietà $\infty^{r-\sigma}$ di classe :

$$
\frac{1}{\alpha_{1} ! \alpha_{2} ! \ldots \alpha_{\tau} !} \sum_{k=0}^{t}(t-k) ! k ! s_{k}\left(\begin{array}{c}
n-\sigma-k \\
t-k
\end{array}\right)\left(\begin{array}{c}
n-\sigma-\rho \\
k
\end{array}\right)
$$

conservando $i$ simboli $\alpha_{\mathrm{j}}(\mathrm{j}=1,2, \ldots, \tau)$ ed $s_{\mathrm{k}}(\mathrm{k}=0,1, \ldots, \mathrm{t}) i$ medesimi signi. ficati che nel n. 1 e nel n. 2.

Di questo teorema era finora noto, in tutta la sua generalità, solo il caso particolare corrispondente all' ipotesi $p=0$.

Come corollario risulta ad osempio che (cfr. n. 1):

Il numero degli iperpiani $\mathrm{r}$-tangenti (ciascuno ad $\mathrm{r}$ rami) della curva $\Gamma_{\mathrm{n}}^{0} \dot{e}$ :

$$
\sum_{k=0}^{r}\left(\begin{array}{c}
n-r-k \\
r-k
\end{array}\right)\left(\begin{array}{c}
n-r-\rho \\
k
\end{array}\right)
$$

4. A risultati di ben maggiore rilievo conduce l'applicazione del teorema generale del n. 2 alla serie lineare descritta dal gruppo degli iperpiani osculatori a $\Gamma_{n}^{0}$ (n. 1) uscenti da un punto variabile in un generico spazio $S_{\sigma}$ di $S_{r}$. Si trova infatti allora che:

Nello spazio $\mathrm{S}_{\mathrm{r}}$ ad $\mathrm{r}$ dimensioni il luogo dei punti tali che da ciascuno $d i$ essi escono almeno, e in generale precisamente, $t$ spazi distinti $\mathrm{S}_{\mathrm{r}-{ }_{\mathrm{yi}}-1}$ $(\mathrm{t} \geq 1 ; i=1,2, \ldots, \mathrm{t})$ osculatori di una data curva razionale $\Gamma_{\mathrm{n}}^{\circ}$ d'ordine $\mathrm{n}$, appartenente ad $\mathrm{s}_{\mathrm{r}}$, con $p$ cuspidi $\left({ }^{(}\right)$e quindi di classe:

$\grave{e}, s e:$

$$
n^{\prime}=r(n-r+1)-(r-1) \rho \text {, }
$$

$$
v_{1}+v_{2}+\ldots+v_{t}=\sigma \leq r,
$$

una varietà di dimensione $\mathrm{r}-\sigma e$ di ordine:

$$
\frac{1}{\alpha_{1} ! \alpha_{2} ! \ldots \alpha_{\tau} !} \sum_{k=0}^{t}(-1)^{k} k !(t-k) ! s_{k}\left(\begin{array}{c}
n-2 r+\sigma-\rho+k-1 \\
k
\end{array}\right)\left(\begin{array}{c}
n^{\prime}-\sigma-k \\
t-k
\end{array}\right) .
$$

Devesi supporre $1 \leq v_{1}<\mathrm{r}$ e $\mathrm{t} \leq \mathbf{n}^{\prime}-\sigma$; mentre ai simboli $\alpha_{j}(\mathbf{j}=1,2, \ldots, \tau)$ $e \mathrm{~s}_{\mathrm{K}}(\mathrm{k}=0,1, \ldots, \mathrm{t})$ vanno attribuiti gli stessi significati che nei $n .1$ e 2. nel n. 1 .

(6) Per la curva $\Gamma_{n}^{0}$ si sottintendano sompre soddisfatte le ulteriori ipotesi precisate

(7) Ritenuto, beninteso, che un iperpiano abbia un contatto di ordine $\mu \geq 1$ con la curva in una sua cuspide $P$ quando contiene l' $S_{\mu}$ (e non soltanto l' $S_{\mu-1}$ ) osculatore in $P$ al ramo $(Q, 1, \ldots, 1)$ di cui $P$ è origine: ofr. nota $\left(^{3}\right)$; cosicchè an iperpiano generico per $P$ non è da considerarsi tangente alla curva in $P$. 
Di questo teorema non era sinora noto, in tutta la sua estensione, neppure il caso particolare relativo all' ipotesi $p=0$.

L'interesse del teorema medesimo si desume dalla possibilita che esso offre senz' altro di risolvere in modo affatto esauriente $i$ due problemi gene. rali seguenti $\left({ }^{(}\right)$:

Essendo $\Gamma$ una curva razionale, anche cuspidata, appartenente ad uno spazio lineare qualunque, determinare:

a) Gli ordini di lutte le varietà multiple di una qualsiasi sviluppabile osculatrice di $\Gamma$.

3) L'ordine della varietà d'intersezione di quante si vogliano sviluppabili osculatrici di $\Gamma$ o loro varietò multiple.

5. Fra le molteplici proposizioni speciali deducibili dal teorema del n. 4 alcune sono assai noteroli. Ad esempio:

Nello spazio $\mathrm{S}_{\mathrm{r}}$ ad $\mathbf{r}$ dimensioni esistono:

$$
\sum_{k=2}^{r}(-1)^{k}\left(\begin{array}{c}
n-r+k-1 \\
k
\end{array}\right)\left(\begin{array}{c}
r n-r^{2}-k \\
r-k
\end{array}\right)
$$

punti caratterizzati dalla proprietà che in ciascun' di essi concorrono $\mathrm{r}$ spazi $\mathrm{S}_{\mathrm{r}-2}$ osculatori distinti di una generica (') curva razionale d'ordine $\mathrm{n}$ appartenente ad $\mathrm{S}_{\mathrm{r}}$.

Giova osservare che, sebbene il numero fornito da quest' ultima proposi. zione sia (per dualità) uguale a quello degli iperpiani $r$-tangenti di una curva razionale $d$ 'ordine $r(n-r+1)$ appartenente ad $S_{r}$, il numero stesso non si poteva ottenere con l'ordinaria formula di $\mathrm{DE}$ JONQUIÈRES: perchè tale curva possiede necessariamente $(r+1)(n-r)$ cuspidi (cfr. n. 1$)$.

Più in generale :

Se $\mu$ è un divisore di $\mathrm{r}$, e $1 \leq \mu \leq \frac{\mathrm{r}}{2}$, esistono in $\mathrm{S}_{\mathrm{r}}$ :

$$
\sum_{k=0}^{r: \mu}(-1)^{k}\left(\begin{array}{c}
n-r+k-1 \\
k
\end{array}\right)\left(\begin{array}{c}
n-r^{2}-k \\
\frac{r}{\mu}-k
\end{array}\right) \mu^{k}
$$

punti da ciascuno dei quali escono $\mathrm{r}: \mu$ spazi distinti $\mathrm{S}_{\mathrm{r}-\mu-1}$ osculatori di una generica (i) curva razionale d'ordine $\mathrm{n}$ appartenente ad $\mathrm{S}_{\mathrm{x}}$.

Ancora :

Supposto $\nu_{1}+\nu_{2}=\mathrm{r}, \varepsilon=1$ se $\nu_{1} \neq \nu_{2}$ ed $\varepsilon=1 / 2$ se $\nu_{1}=v_{2}$, una curva

(8) Vedasi l'altro mio lavoro pure in corso di stampa nei "Commentarii math. Helve. ici : A. Longer, Sulle suiluppabili asculatrici de le curve razionali iperspaziali. 
razionale $\Gamma_{\mathrm{n}}^{0}$ d'ordine $\mathrm{n}$, appartenente ad $\mathrm{S}_{\mathrm{r}}$ e con $\mathrm{\rho}$ cuspidi $\left({ }^{6}\right)$, ammette:

$$
2 \varepsilon v_{1} \nu_{2}(n-r-p+1)+\varepsilon r(n-r-1) \rho-2 \varepsilon(r-1)\left(\begin{array}{l}
\rho \\
2
\end{array}\right)
$$

spazi $\mathrm{S}_{\mathrm{r}-2}$ aventi ciascuno con essa due distinti contatti rispettivamente $v_{1}$-punto e $v_{2}$-punto.

In particolare, quando $\rho=0$ il risultato concorda con quello offerto da note formule del SEvERI $(\%)$.

(9) F. Severi, I gruppi neutri con elementi multipli in un'involuzione sopra un ente razionale, *Rendiconti dell'Accademia dei Lincei », 9, 1900,

F. SeverI, Sopra alcune singolarità delle curve di un iperspazio, "Memorie dell'Aecademia di Torino, 51, 1902, n. 7. 